\title{
Adapting to heterogeneous comfort levels
}

\author{
Luca De Sanctis $\dagger$ and Tobias Galla†t \\ $\dagger$ The Abdus Salam International Center for Theoretical Physics, Strada Costiera 11, \\ 34014 Trieste, Italy \\ $\ddagger I N F M-C N R$, Trieste-SISSA Unit, V. Beirut 2-4, 34014 Trieste, Italy
}

\begin{abstract}
We study the learning dynamics of agents who adapt to heterogeneous comfort levels in the context of an El-Farol type game, and show that even an infinitesimal degree of heterogeneity in the resource levels leads to a significant reduction of the fluctuations of the collective action, and removes the phase transition observed in models with homogeneous comfort level. Our analysis is based on dynamical methods of disordered systems theory, in particular on a generating functional approach, and confirmed by numerical experiments. We also report on simulations of a system in which the comfort levels fluctuate in time, and point out crucial differences between models in which the comfort levels of the agents fluctuate collectively and individually respectively. Finally we comment on a possible characterisation of El-Farol and Minority Games according to the presence or absence of ergodicity-breaking phase transitions at infinite integrated response.
\end{abstract}

PACS numbers: 02.50.Le, 87.23.Ge, 05.70.Ln, 64.60.Ht

E-mail: lde_sanc@ictp.it, galla@ictp.it

\section{Introduction}

Complex adaptive systems of inductive agents often display a remarkably rich global behaviour, which cannot be explained straightforwardly from their microscopic interactions. The tools of statistical mechanics have here been seen to be able to provide further insight, and allow for theoretical progress for a variety of agent-based models. Most notably, analytical solutions for the Minority Game (MG), a mathematical abstraction of the El-Farol bar problem, have been obtained using equilibrium and nonequilibrium techniques of disordered systems theory [1, 2, 3.

In the El-Farol bar problem [4, $N=100$ customers have to decide independently whether or not to attend a concert in the bar, the latter having a capacity of $\lambda=60$ seats. Agents will in general not enjoy the bar if it is too crowded, i.e. if more than $\lambda$ agents attend. Thus depending on the attendance $A$, agents who go to the bar are considered winners if $A<\lambda$, and conversely, agents who decide to stay home win if $A>\lambda$. We will refer to $\lambda$ as the comfort level in the following. Players in the El-Farol bar problem are inductive agents: they use individual 'predictors', based on the past attendance, to predict whether or not the bar will be crowded at the next time step, 
and according to these predictions they then decide whether to attend or not. They learn by experience and employ the most accurate predictor which they can access.

Under fairly weak assumptions it can be shown that the temporal average of the attendance, $\langle A\rangle$, converges to the comfort level $\lambda$ in Arthur's El-Farol model provided the predictors are not systematically biased [4. A second question relates to the degree to which agents are able to reduce the fluctuations of $A$ about $\lambda$, i.e. to the quantity $\sigma^{2}=\left\langle A^{2}\right\rangle-\langle A\rangle^{2}$, where the brackets denote an average over time. We will refer to $\sigma^{2}$ as the volatility in the following.

The MG in its original form [5] is a mathematical formulation of the El-Farol problem at comfort level $\lambda=N / 2$. All agents take binary decisions at each time-step, and players in the minority group win. Predictors, fixed at the beginning of the game and randomly generated, are here unbiased in the sense that they advise to attend or stay home with equal statistical weights. Generalisations to $\lambda \neq N / 2$ and/or systematically biased strategies are possible, and have been studied by numerical simulations in [6, 7]. More systematic studies of El-Farol games with general uniform comfort levels have been presented [8], see also [9].

In this paper we consider an El-Farol type problem with heterogeneous comfort levels, so that each agent $i$ prefers to attend the bar only if $A<\lambda_{i}$, where $\lambda_{i}$ varies across the population of agents. Our work is an extension of [8], and we reproduce some results reported there as a special case. We here employ a dynamical approach whereas the analysis of [8] is based on static replica calculations. Both approaches are now standard in the context of MGs and related models [1, 2].

The purpose of this study is here twofold. Firstly we aim at understanding the role of heterogeneous comfort levels for the learning dynamics of the agents. In particular we will be concerned with the question of whether or not heterogeneity in the comfort levels compromises the ability of the population of agents to converge to a 'mean' comfort level. Similarly we will study the influence of heterogeneity in the comfort levels on the temporal fluctuations of the attendance. The second reason for studying the present system rests in more theoretical issues related to the phase behaviour of MGs and related models. In the standard MG a phase transition between an ergodic and a non-ergodic regime has been found, and identified with a point in parameter space in which the integral over the response function of the system becomes singular [2, 3]. In the nonergodic regime a continuum of attractors of the learning dynamics appears to be present and introduces interesting effects such as a marked sensitivity to initial conditions, which is absent in the ergodic phase.

Some variants of the MG, however, which are typically only slight modifications of the original game have been seen not to display the above transition. Some variants exhibit no transition at all, others one that is of a different type than the one described above. The transition is absent for example in so-called grand-canonical MGs (GCMG) [10], and similarly such an absence may be suspected for MG models with finite score memories [11] although no analytical results are available as yet. In MGs with impact correction [12] and dilution of the agents' interaction matrix [13] the transition of the 
type as in the original MG is preceded by one which is referred to as 'memory-onset' transition [2]. Ergodicity breaking here still occurs, but at finite integrated response. This type of transition has been associated with replica-symmetry breaking in static studies. The second purpose of this paper is thus to try to shed some more light on the circumstances under which MG models display a transition of the type of the original MG. We here show that MGs with any degree of heterogeneity in the comfort levels do not display this type of transition, and conclude with some conjectures on how the structure of the agents' learning dynamics may determine the presence or otherwise of such a transition.

\section{Definition of the model}

We consider a system of $N$ agents, labelled with Roman indices $i, j \in\{1, \ldots, N\}$. At each round $t$ of the game each agent $i$ takes a binary decision $b_{i}(t) \in\{-1,1\}$ in response to the observation of a common piece of publicly available information, labelled by $\mu(t)$. $b_{i}(t)=1$ may here correspond to player $i$ attending the bar at time $t$, and $b_{i}(t)=-1$ to him not attending. While the information encodes the actual history (i.e. the previous attendances) in the original version of the El-Farol problem and in the original MG, we will here assume that $\mu(t)$ is chosen randomly and independently from a set with $P=\alpha N$ possible values at any $t$, i.e. $\mu(t) \in\{1, \ldots, \alpha N\}$. This has been seen not to alter the qualitative behaviour of different variants of the MG [1, 2]. $\alpha=P / N$ is here the main control parameter of the model, and taken not to scale with $N$. One then defines the re-scaled total outcome at round $t$ as $A(t)=N^{-1 / 2} \sum_{i} b_{i}(t)$, resulting in $-\sqrt{N} \leq A(t) \leq \sqrt{N}$ (in steps of $2 / \sqrt{N})$. Note that in this notation $\left(b_{i}(t) \in\{-1,1\}\right.$ as opposed to $b_{i}(t) \in\{0,1\}$ for not-attending/attending the bar) an attendance of $N / 2$ in the original El-Farol problem corresponds to $A=0$, and accordingly the comfort level in an actual MG (in which the bar is considered crowded if more than precisely half of the agents attends) would be $\lambda=0$. Agents who prefer a bar filled by less than half have $\lambda_{i}<0$, agents who can enjoy the bar even if it is populated by more than $N / 2$ attendees have $\lambda_{i}>0$. In general the attendance in the original El-Farol problem can be obtained as $(\sqrt{N} A(t)+N) / 2$ from the global action $A(t)$, in our conventions. Having this simple linear transformation in mind, we will refer to $A(t)$ as the attendance at time $t$ in the following, and, as mentioned above, to $\lambda_{i}$ as the comfort level of player $i$. Note that $A(t)$ remains a well-defined finite quantity in the thermodynamic limit $N \rightarrow \infty$ at $\alpha=P / N$ finite, with which the statistical mechanics analysis of the model is concerned.

In order to take their decisions on whether or not to attend each agent $i$ holds two fixed trading strategies (look-up tables) $\mathbf{R}_{i, a}=\left(R_{i, a}^{1}, \ldots, R_{i, a}^{P}\right) \in\{-1,1\}^{P}$, with $a= \pm 1$. The binary entries $R_{i, a}^{\mu}$ are drawn from some distribution (to be specified below) before the start of the game; these entries (along with the comfort levels $\lambda_{i}$ ) represent the quenched disorder of this problem. If agent $i$ decides to use strategy $a$ in round $t$ of the game, his action at this stage will be $b_{i}(t)=R_{i, a}^{\mu(t)}$. The agents decide which strategy to use based on points $p_{i, a}(t)$ which they allocate to each of their strategies. These virtual 
scores are based on the player's potential success had he always played that particular strategy, and are updated as follows at every time-step:

$$
p_{i, a}(t+1)=p_{i, a}(t)-R_{i, a}^{\mu(t)}\left(A(t)-\lambda_{i}\right) .
$$

Strategies which would have produced a decision to the liking of player $i$ are thus rewarded, i.e. if $A(t)>\lambda_{i}$ the scores of strategies $a$ with $R_{i, a}^{\mu(t)}<0$ are increased and vice versa for $A(t)<\lambda_{i}$. In the MG literature the above type of process (11) with an explicit dependence on the influx of information patterns $\mu(t)$ is generally referred to as 'on-line dynamics'. At each round $t$ each player $i$ then uses the strategy in his arsenal with the highest score, i.e. $b_{i}(t)=R_{i, s_{i}(t)}^{\mu(t)}$, where $s_{i}(t)=\arg \max _{a} p_{i, a}(t)$. It remains to specify the statistics of the $\left\{R_{i, a}^{\mu}\right\}$. We here choose

$$
P\left(R_{i, a}^{\mu}\right)=\frac{1+\rho_{i} / \sqrt{N}}{2} \delta_{R_{i, a}^{\mu}, 1}+\frac{1-\rho_{i} / \sqrt{N}}{2} \delta_{R_{i, a}^{\mu},-1},
$$

as the distribution from which the $R_{i, a}^{\mu}$ are drawn (with $\delta_{x, y}=1$ if $x=y$ and $\delta_{x, y}=0$ otherwise), so that $\overline{R_{i, a}^{\mu}}=\rho_{i} / \sqrt{N}$ for all $a, \mu(\bar{\cdots}$ here denotes an average over the quenched disorder). In the limit of large $N$, which we will eventually consider, the distribution of the $R_{i, a}^{\mu}$ is well-defined for any $\rho_{i} \in \mathbb{R}$. If $\rho_{i}>0$ then player $i$ 's strategies are more likely to advise him to take a positive action $\left(b_{i}=+1\right)$ than a negative one, and vice versa. We will refer to $i$ 's strategies as being consistent with his comfort level $\lambda_{i}$ if $\rho_{i}=\lambda_{i}$, and as inconsistent otherwise. For an agent with consistent strategies the probability of him attending the bar upon randomly choosing one of his strategies (regardless of their scores) corresponds to his comfort level. The scaling of the comfort levels and strategy biases with $N$ has here been chosen to ensure a well-defined thermodynamic limit, and corresponds to the choices of 8 .

In the following we will consider a population of agents in which all $\rho_{i}$ are drawn independently from a fixed distribution $Q(\rho)$, and the $\lambda_{i}$ from $R(\lambda)$. The standard MG is recovered for $\lambda_{i}=\rho_{i} \equiv 0 \forall i$, i.e. $Q(\rho)=\delta(\rho)$ and $R(\lambda)=\delta(\lambda)$. As we will see in the further analysis, the specific details of the distribution $Q(\rho)$ are unimportant, and the only feature relevant for the properties of the model is the mean strategy bias $\rho_{0}=\int d \rho Q(\rho) \rho$. We will also demonstrate that the model is invariant against simultaneous uniform shifts of the $\rho_{i}$ and $\lambda_{i}$, i.e. under $\lambda_{i} \rightarrow \lambda_{i}+\Delta$ and $\rho_{i} \rightarrow \rho_{i}+\Delta$ for any $\Delta \in \mathbb{R}$. Without loss of generality we will therefore mostly restrict to cases with $\rho_{0}=0$ in the following.

Finally the key observables we will study in this model are given by the deviation of the mean attendance from the mean comfort level $\lambda_{0}=\int d \lambda R(\lambda) \lambda$, i.e. by $\left|\langle A\rangle-\lambda_{0}\right|$, and by the fluctuations of the attendance about its mean, i.e. by $\sigma^{2}=\left\langle A^{2}\right\rangle-\langle A\rangle^{2}$. $\langle\cdots\rangle$ here stands for an average over time in potential stationary states of the system, i.e. after some sufficiently long equilibration time. To conclude this section we note that that the model of [8] can be recovered upon assuming homogeneous comfort levels and strategy biases, i.e. by setting $\rho_{i} \equiv \rho_{0}$ and $\lambda_{i} \equiv \lambda_{0}$ for all $i$. 


\section{Statistical mechanics analysis}

We will here pursue a dynamical approach based on a generating functional analysis of the score update rules. We here consider the so-called batch process of the learning dynamics, which for the present case reads

$$
q_{i}(t+1)=q_{i}(t)-\frac{2}{\sqrt{N}} \sum_{\mu} \xi_{i}^{\mu}\left[\frac{1}{\sqrt{N}} \sum_{j}\left\{\xi_{j}^{\mu} s_{j}(t)+\omega_{j}^{\mu}\right\}-\lambda_{i}\right]+h_{\lambda_{i}}(t),(3)
$$

similar to that of the standard MG 14, 2]. We have here introduced the score differences $q_{i}(t)=\frac{1}{2}\left(p_{i, 1}(t)-p_{i,-1}(t)\right)$ and the quantities $\xi_{i}^{\mu}=\frac{1}{2}\left(R_{i, 1}^{\mu}-R_{i,-1}^{\mu}\right)$ and $\omega_{i}^{\mu}=\frac{1}{2}\left(R_{i, 1}^{\mu}+R_{i,-1}^{\mu}\right)$, using conventions which are now standard in the MG-literature. $s_{j}(t) \in\{-1,+1\}$ indicates the strategy player $j$ is using at time $t$ and is given by $s_{j}(t)=\operatorname{sgn}\left[q_{j}(t)\right]$, so that player $j$ 's action at time $t$ reads $\omega_{j}^{\mu}+\xi_{j}^{\mu} s_{j}(t)$ on the occurrence of information pattern $\mu$. $h_{\lambda_{i}}(t)$ is an external perturbation field, which is set to zero in all simulations and mostly a mathematical device added to generate response functions. Perturbations are assumed to be identical for agents with identical comfort level, hence the subscript $\lambda_{i}$. Note also that compared to the on-line process an effective average over all information patterns $\mu$ has been performed at each time-step and that time has been re-scaled.

The above batch process is the starting point for the dynamical analysis of the problem based on generating functionals. This technique is now standard in the context of the MG and we will only report the final outcome of the theory here, with some additional information in the appendix. For the further mathematical details of the computation in similar cases we refer to the recent textbook [2].

The generating functional analysis turns the Markovian problem of interacting agents into a self-consistent description in terms of decoupled effective agents subject to non-Markovian stochastic processes, and is exact in the thermodynamic limit $N \rightarrow \infty$ (at fixed $\alpha=P / N$ ). For an effective agent with strategy bias $\lambda$ this process reads

$$
q_{\lambda}(t+1)=q_{\lambda}(t)-\alpha \sum_{t^{\prime} \leq t}(\mathbf{I}+G)_{t t^{\prime}}^{-1} s_{\lambda}\left(t^{\prime}\right)+\sqrt{\alpha} \eta_{\lambda}(t)+h_{\lambda}(t)
$$

where the second term on the right-hand side marks a retarded interaction in time and renders the process non-Markovian. $s_{\lambda}(t)$ is given by $s_{\lambda}(t)=\operatorname{sgn}\left[q_{\lambda}(t)\right]$ and $\eta_{\lambda}(t)$ represents the stochasticity of this process. More precisely, $\eta_{\lambda}(t)$ is Gaussian noise of zero mean, and with temporal correlations

$$
\left\langle\eta_{\lambda}(t) \eta_{\lambda}\left(t^{\prime}\right)\right\rangle_{\star}=\left[(\mathbb{I}+G)^{-1} D\left(\mathbb{I}+G^{T}\right)^{-1}\right]_{t t^{\prime}}+2 f_{t} f_{t^{\prime}}-2 \lambda\left(f_{t}+f_{t^{\prime}}\right)+2 \lambda^{2} E_{t t^{\prime}}
$$

Here $\mathbf{I}_{t t^{\prime}}=\delta_{t t^{\prime}}$ is the identity matrix and $E_{t t^{\prime}}=1 \forall t, t^{\prime}$, and $\langle\cdots\rangle_{\star}$ refers to an average over realisations of the effective process (i.e. over realisations of the $\eta_{\lambda}$ ). $C$ and $G$ are the correlation and response functions of the system respectively

$$
C_{t t^{\prime}}=\lim _{N \rightarrow \infty} N^{-1} \sum_{i} \overline{\left\langle\left\langle s_{i}(t) s_{i}\left(t^{\prime}\right)\right\rangle\right\rangle}, \quad G_{t t^{\prime}}=\lim _{N \rightarrow \infty} N^{-1} \sum_{i} \frac{\partial \overline{\left.\left\langle s_{i}(t)\right\rangle\right\rangle}}{\partial h_{\lambda_{i}}\left(t^{\prime}\right)}
$$


with $\langle\langle\cdots\rangle\rangle$ an average over potentially random initial conditions from which the dynamics is started. The matrix $D$ finally is given by $D_{t t^{\prime}}=1+C_{t t^{\prime}}$ for all $t, t^{\prime}$ and we have

$$
f_{t}=\sum_{t^{\prime}}\left[(\mathbb{I}+G)^{-1}\left(\rho_{0} \mathbb{I}+G^{\prime}\right)\right]_{t t^{\prime}}
$$

with

$$
G_{t t^{\prime}}^{\prime}=\lim _{N \rightarrow \infty} N^{-1} \sum_{i} \lambda_{i} \frac{\partial \overline{\left\langle\left\langle s_{i}(t)\right\rangle\right\rangle}}{\partial h_{\lambda_{i}}\left(t^{\prime}\right)} .
$$

These order parameters are then to be determined as averages over realisations of the effective processes and over the distribution of $\lambda$

$$
\begin{aligned}
& C_{t t^{\prime}}=\int d \lambda R(\lambda)\left\langle s_{\lambda}(t) s_{\lambda}\left(t^{\prime}\right) \mid \lambda\right\rangle_{\star} \\
& G_{t t^{\prime}}=\int d \lambda R(\lambda) \frac{\partial}{\partial h_{\lambda}\left(t^{\prime}\right)}\left\langle s_{\lambda}(t) \mid \lambda\right\rangle_{\star} \\
& G_{t t^{\prime}}^{\prime}=\int d \lambda R(\lambda) \lambda \frac{\partial}{\partial h_{\lambda}\left(t^{\prime}\right)}\left\langle s_{\lambda}(t) \mid \lambda\right\rangle_{\star}
\end{aligned}
$$

(where $\langle\cdots \mid \lambda\rangle_{\star}$ is an average over realisations of the effective process restricted to representative agents with comfort level $\lambda$ ). The further analysis then assumes the existence of an ergodic stationary state (in which correlation and response functions depend only on time-differences, i.e. $C_{t t^{\prime}}=C\left(t-t^{\prime}\right)$ and similarly for $G$ and $G^{\prime}$ and in which the integrated responses remain finite) and proceeds along the lines of [14. We will not report these further steps here, but only quote some of the resulting equations describing the persistent order parameters in the appendix. These timeindependent order parameters are in the present problem given by the persistent part $c=\lim _{T \rightarrow \infty} T^{-1} \sum_{\tau \leq T} C(\tau)$ of the correlation function and by the integrated responses $\chi=\sum_{\tau} G(\tau)$ and $\chi^{\prime}=\sum_{\tau} G^{\prime}(\tau)$.

The two observables which we will focus on, namely the mean attendance and the fluctuations of the attendance, can be computed from the effective particle problem as follows. Similarly to [14] one finds that

$$
\overline{\left\langle\left\langle A_{t}\right\rangle\right\rangle}=f_{t}, \quad \overline{\left\langle\left\langle A_{t} A_{t^{\prime}}\right\rangle\right\rangle}=\frac{1}{2}\left[(\mathbf{I}+G)^{-1} D\left(\mathbf{I}+G^{T}\right)^{-1}\right]_{t t^{\prime}}+f_{t} f_{t^{\prime}}
$$

resulting in $\ddagger$

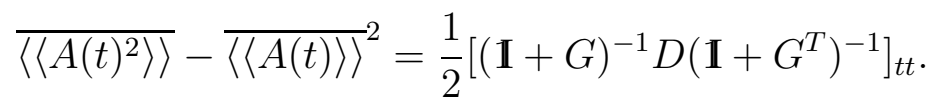

Temporal averages can then be expressed in terms of persistent order parameters, and we have

$$
\langle A\rangle=\frac{\rho_{0}+\chi^{\prime}}{1+\chi}, \quad \sigma^{2}=\frac{1}{2}\left[\frac{1+c}{1+\chi}+1-c\right]
$$

The former relation is exact, the second obtained within a common approximation following the lines of [2].

$\ddagger$ Note here also that $\left\langle\eta_{\lambda}(t) \eta_{\lambda}\left(t^{\prime}\right)\right\rangle_{\star}=2 \overline{\left\langle\left\langle\left(A_{t}-\lambda\right)\left(A_{t^{\prime}}-\lambda\right)\right\rangle\right\rangle}$, reflecting the term $A(t)-\lambda_{i}$ in the update rules of the score difference of player $i$. 


\section{Results}

One observes from the above effective agent problem that the distribution of strategy biases $Q(\rho)$ enters only through its first moment $\rho_{0}$ as claimed above. Furthermore it is straightforward to check on the level of the effective process that simultaneous shifts of the distributions of $\rho$ and $\lambda$ (i.e. $\rho_{i} \rightarrow \rho_{i}+\Delta \forall i$ at the same time as $\lambda_{i} \rightarrow \lambda_{i}+\Delta$ with some $i$-independent $\Delta \in \mathbb{R}$ ) do not effect the transients or stationary states of the model, and it becomes clear that strategy biases and comfort levels are fully equivalent in the homogeneous case as already found in [8]. This is also verified in simulations. Thus we can concentrate on the case $\rho_{0}=0$.

\subsection{Heterogeneous comfort levels at consistent strategies}

We here impose consistency of the strategy biases with the individual comfort levels, i.e. that $\rho_{i}=\lambda_{i}$ for all $i$. If the $\lambda_{i}$ are then homogeneous over the population of agents, i.e. if $\lambda_{i}=\rho_{i} \equiv \rho_{0}$ for all $i$, then the problem can be seen to be equivalent to the standard MG with random external information [8]. Due to the invariance under uniform shifts in the $\rho_{i}$ and $\lambda_{i}$ this equivalence of the model with homogeneous consistent strategies to the MG holds irrespectively of $\rho_{0}$.

We now turn to El-Farol games with a heterogeneous distribution of the $\lambda_{i}$ and $\rho_{i}$, still maintaining consistency $\lambda_{i}=\rho_{i}$. We here choose the simplest possible case, namely a bi-modal distribution

$$
R(\lambda)=\frac{1}{2}[\delta(\lambda-\varepsilon)+\delta(\lambda+\varepsilon)]
$$

to study the effects of heterogeneity in the comfort levels on the behaviour of the model, with $\varepsilon>0$ a model parameter measuring the degree of heterogeneity. $\varepsilon=0$ reproduces the standard MG.

One first realises that the mean attendance $\langle A\rangle$ is given by the mean comfort level $\lambda_{0}=\int d \lambda R(\lambda) \lambda=0$. In the theoretical analysis this is easily seen to be the case as $\chi^{\prime}=0$ due to symmetry with respect to $\varepsilon \leftrightarrow-\varepsilon$, and is also confirmed in simulations (not shown). The magnitude of the fluctuations of the attendance about this level is shown in Fig. 1. both as a function of $\alpha$ at fixed $\varepsilon$ (left) and as a function of $\varepsilon$ at fixed $\alpha$ (right). For $\varepsilon=0$ one finds the functional dependence of $\sigma^{2}$ on $\alpha$ typical of the well-known MG, with a minimum attained at an intermediate $\alpha_{c} \approx 0.33$ and both highvolatility and low-volatility branches at $\alpha<\alpha_{c}$ depending on initial conditions, as shown in the left panel of Fig. 1 for comparison, with open circles marking unbiased starts $\left(q_{i}(0)=0\right)$, and filled circles starts from strongly biased initial conditions $\left(\left|q_{i}(0)\right| \gg 1\right)$. One finds that $\sigma^{2}$ diverges as $\alpha \rightarrow 0$ for unbiased starts, and that $\sigma^{2} \rightarrow 0$ for strongly biases initial conditions [2, 3]. For $\alpha>\alpha_{c}$ the starting point of the dynamics has no influence on the persistent order parameters in the stationary state. In the theoretical analysis the phase transition point separating both regimes is identified as the point at which the integrated response $\chi$ diverges, marking the breakdown of the assumptions regarding ergodicity. 

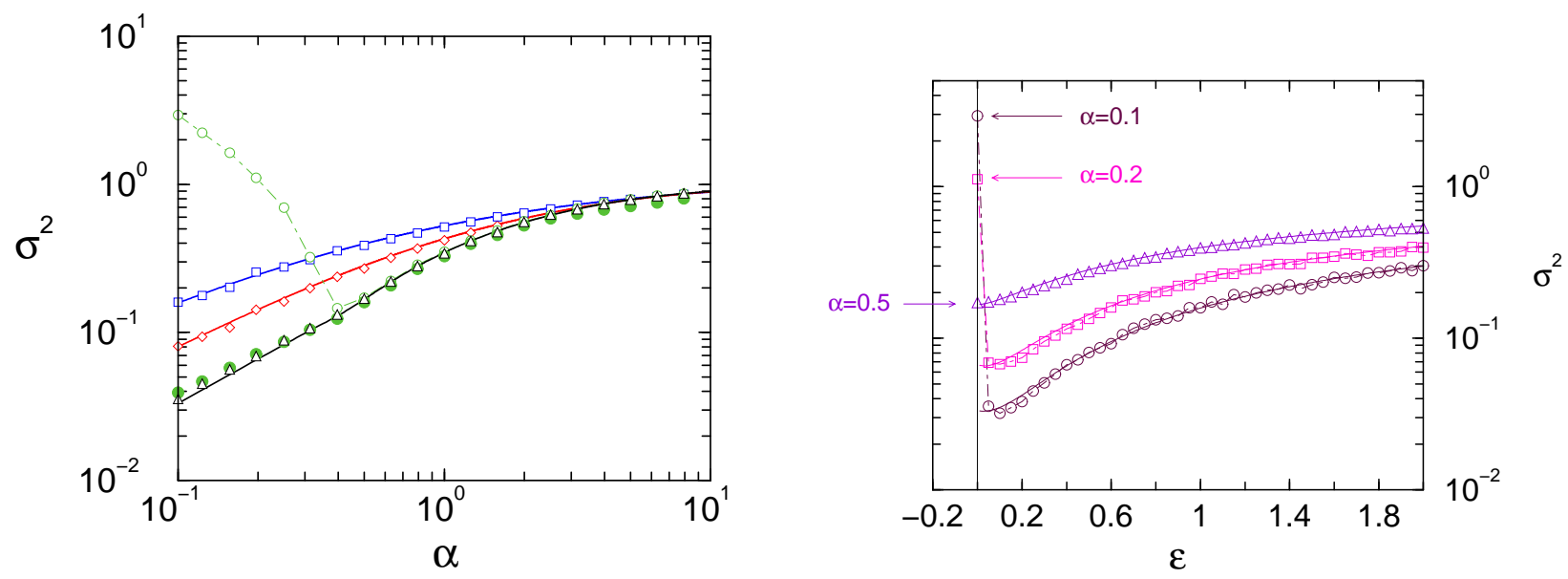

Figure 1. (Colour on-line) Volatility for the game with consistent strategies $\left(\rho_{i}=\right.$ $\left.\lambda_{i} \forall i\right)$ and bi-modal distribution $R(\lambda)=[\delta(\lambda+\varepsilon)+\delta(\lambda-\varepsilon)] / 2$. Left: $\sigma^{2}$ as a function of $\alpha$ for different $\varepsilon=0$ (circles), $\varepsilon=1$ (squares), $\varepsilon=0.5$ (diamonds) and $\varepsilon=0.01$ (triangles). Open symbols are from simulations of the corresponding on-line games started from tabula rasa initial conditions $q_{i}(0)=0$ (with $N=300$ agents, averages over 50 samples are taken, simulations run for 50000 on-line steps (or more for small $\varepsilon)$ ). Solid lines are from the analytical theory. Filled circles are for the standard MG $(\varepsilon=0)$ from strongly biased initial conditions. Right: $\sigma^{2}$ as a function of $\varepsilon$ at fixed $\alpha=0.1,0.2,0.5$ (circles, squares, triangles respectively). Symbols are simulations of the on-line process started from tabula rasa initial conditions, lines the analytical theory in the ergodic phase. Arrows point out that there are discontinuities of $\sigma^{2}$ at $\varepsilon \rightarrow 0$ for $\alpha<\alpha_{c}$ but not at $\alpha=0.5>\alpha_{c}$.

The behaviour of the model with heterogeneous (but consistent) comfort levels is fundamentally different. For $\varepsilon>0$ all curves $\sigma^{2}$ are found to be increasing functions of $\alpha$, and the volatility remains low at low values of $\alpha$ (for all values of $\varepsilon$ tested here), as shown in the left panel of Fig. 1. We here depict measurements from simulations (markers) as well as the predictions of the analytical theory (lines), and find excellent agreement. No significant dependence on initial conditions is found. The theoretical analysis shows no sign of a divergence of either $\chi$ or $\chi^{\prime}$ so that we conclude that the ergodicity-broken phase of the standard MG is absent as soon as any degree of heterogeneity is added to the comfort levels. We have also performed an analysis along the lines of [15] to check for a breakdown of weak long-term memory assumptions at finite integrated response, and could find no such a memory-onset transition. Thus the system is fully ergodic at all $\alpha>0$ for $\varepsilon>0$.

The marked difference between the models with and without heterogeneity in the comfort levels is demonstrated in plots of $\sigma^{2}$ versus $\varepsilon$ at different values of $\alpha$ in the right panel of Fig. 1. For $\alpha>\alpha_{c}$ one observes a smooth dependence of $\sigma^{2}$ on $\varepsilon$ with no discontinuity at $\varepsilon \rightarrow 0$. For $\alpha<\alpha_{c}$ however, a characteristic jump occurs at $\varepsilon \rightarrow 0$ if simulations from zero initial conditions are considered. For all $\varepsilon \neq 0 \sigma^{2}$ remains low, whereas a substantially higher volatility is found at $\varepsilon=0$. The magnitude of the jump 


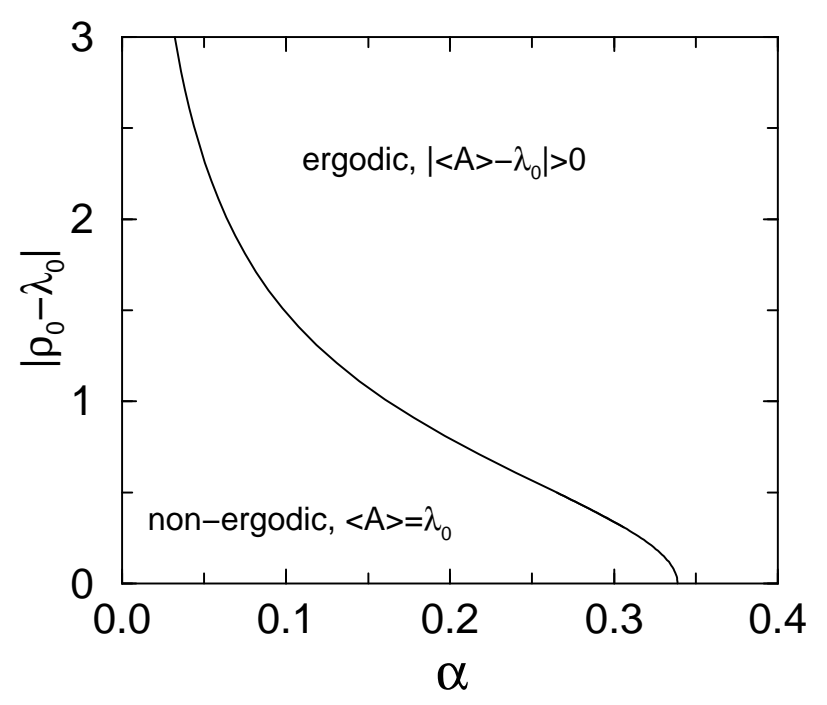

Figure 2. Phase diagram of the model with homogeneous resource level and homogeneous strategy bias $\left(\rho_{i} \equiv \rho_{0}\right.$ and $\lambda_{i} \equiv \lambda_{0}$ for all $\left.i\right)$ in the $\left(\alpha,\left|\rho_{0}-\lambda_{0}\right|\right)$ plane. Up to re-scaling the diagram is identical to that found in [8] by static methods.

here depends on the choice of $\alpha<\alpha_{c}$ an increases as $\alpha$ becomes smaller. Note that a similar behaviour has been found in MGs with impact correction or dilution 1, 2, 13.

Finally, while we have presented results only for bimodal distributions of the $\lambda_{i}$ we note that the theory presented here straightforwardly applies to more general distributions $R(\lambda)$ as well. Simulations of the model with Gaussian $R(\lambda)$ of different non-zero widths $\varepsilon$ demonstrate that the phase transition is absent also in this case and that qualitative behaviour of the model is then similar to that of the game with a bimodal distribution of the $\lambda_{i}$. Thus the specific distribution of the comfort levels seems to be irrelevant as far as the absence of the phase transition is concerned, and the only relevant factor appears to be the presence or otherwise of any type of heterogeneity.

\subsection{Inconsistent strategies}

We here consider the case in which $\lambda_{i} \neq \rho_{i}$. The case of uniformly inconsistent strategies has been studied in [8]. In our notation the results of [8] correspond to choosing $\rho_{i} \equiv \rho_{0}$ and $\lambda_{i} \equiv \lambda_{0}$ for all $i$, where $\left|\lambda_{0}-\rho_{0}\right|$ measures the degree of inconsistency. The resulting phase diagram is shown in Fig. 2, and corresponds to that derived in [8] by different methods. The dependence only on the combination $\left|\lambda_{0}-\rho_{0}\right|$ reflects the equivalence of strategy biases and comfort levels in the homogeneous case. Without loss of generality, we will (mostly) consider the case $\rho_{0}=0$. At any $\lambda_{0}$ one then finds a phase transition of the type which is observed in the standard MG, with an ergodic phase at $\alpha \geq \alpha_{c}\left(\lambda_{0}\right)$, and non-ergodic behaviour below $\alpha_{c}$. In the ergodic regime time-translation invariance is maintained and the integrated response $\chi$ remains finite. The phase transition line in Fig. 2 is marked by $\chi \rightarrow \infty$ (note that in the case of homogeneous strategy biases $\chi^{\prime}$ is 
a multiple of $\chi$ so that a divergence of $\chi^{\prime}$ is equivalent to one of $\chi$ ).

It is here interesting to study the convergence of the mean attendance $\langle A\rangle$ to the mean comfort level $\lambda_{0}$. As shown in [8], one finds that indeed $\langle A\rangle=\lambda_{0}$ below $\alpha_{c}$, but not above (if $\lambda_{0} \neq 0$, for $\lambda_{0}=0$ one always has $\langle A\rangle=0$ by symmetry). In other words at fixed $\alpha$ agents are able to recover the mean comfort level even in the inconsistent case, provided strategy biases and comfort levels are uniform and the inconsistency smaller than some critical value $\lambda_{0 c}(\alpha)$. This is illustrated in the left panel of Fig. [3 (lower curves). At the same time the transition between the phases in which the mean comfort level can be retrieved, and the one where it cannot, coincides with the ergodicity-breaking transition. At fixed $\lambda_{0}$ the system is ergodic for $\alpha>\alpha_{c}\left(\lambda_{0}\right)$, and non-ergodic for $\alpha<\alpha_{c}\left(\lambda_{0}\right)$, with large volatilities for unbiased starts at low $\alpha$, and small volatilities for biased starts below the transition. Measurements of the volatility for unbiased starts are shown in Fig. 3 (right panel, upper curves).

We now again turn to the effects of heterogeneity on this system. In the present case of inconsistent strategies one has to distinguish heterogeneities in the comfort levels $\lambda_{i}$ from those in the strategy biases $\rho_{i}$. As we will see below strategy biases and comfort levels are no longer equivalent in the heterogeneous case, and it makes a crucial difference whether heterogeneities are added to one or the other. In order to disentangle the effects of both we study the following cases:

(i) heterogeneous strategy biases at homogeneous comfort level $\left(\rho_{i}= \pm 0.05\right.$ randomly and $\lambda_{i} \equiv 0.5$, diamonds in Fig. 31)

(ii) homogeneous strategy biases at heterogeneous comfort levels $\left(\rho_{i} \equiv 0\right.$ and $\lambda_{i}=$ $0.5 \pm 0.05$, circles in Fig. (3)

(iii) strategy biases and comfort levels both heterogeneous $\left(\rho_{i}= \pm 0.05\right.$ and $\lambda_{i}=$ $0.5 \pm 0.05$ with no correlation between $\rho_{i}$ and $\lambda_{i}$, squares in Fig. 3)

(iv) strategy bias and comfort level both homogeneous $\left(\rho_{i} \equiv 0, \lambda_{i} \equiv 0.5\right.$, triangles in Fig. 3)

(iv) is the case of homogeneous comfort levels and strategy biases as discussed above. One finds that the transition as observed in (iv) is preserved only in case (i) where comfort levels are homogeneous, but that it is absent in cases (ii) and (iii) where comfort levels are heterogeneous. We illustrate this by plotting the mean attendance in the stationary states for all cases in the left panel of Fig. 3. While the mean comfort level $\lambda_{0}=0.5$ is successfully retrieved at low $\alpha$ in (i), systematic deviations are present irrespectively of $\alpha$ in (ii) and (iii). The right panel of the figure demonstrates that the volatility remains low in cases (ii) and (iii) (heterogeneous comfort levels) at low $\alpha$ for unbiased starts whereas $\sigma^{2}$ diverges as $\alpha \rightarrow 0$ for the systems (i) and (iv) where the comfort level is uniform. Note also the good agreement of the numerical experiments with the analytical theory in the ergodic regimes, up to small deviations due to finitesize and equilibration effects. Finally we also report simulations of a system with homogeneous comfort levels and strategy biases, but shifted by an amount $\Delta=-0.5$ with respect to (iv), i.e. we choose $\rho_{i} \equiv \rho_{0}=-0.5$ and $\lambda_{i} \equiv \lambda_{0}=0$ for all $i$ (stars 

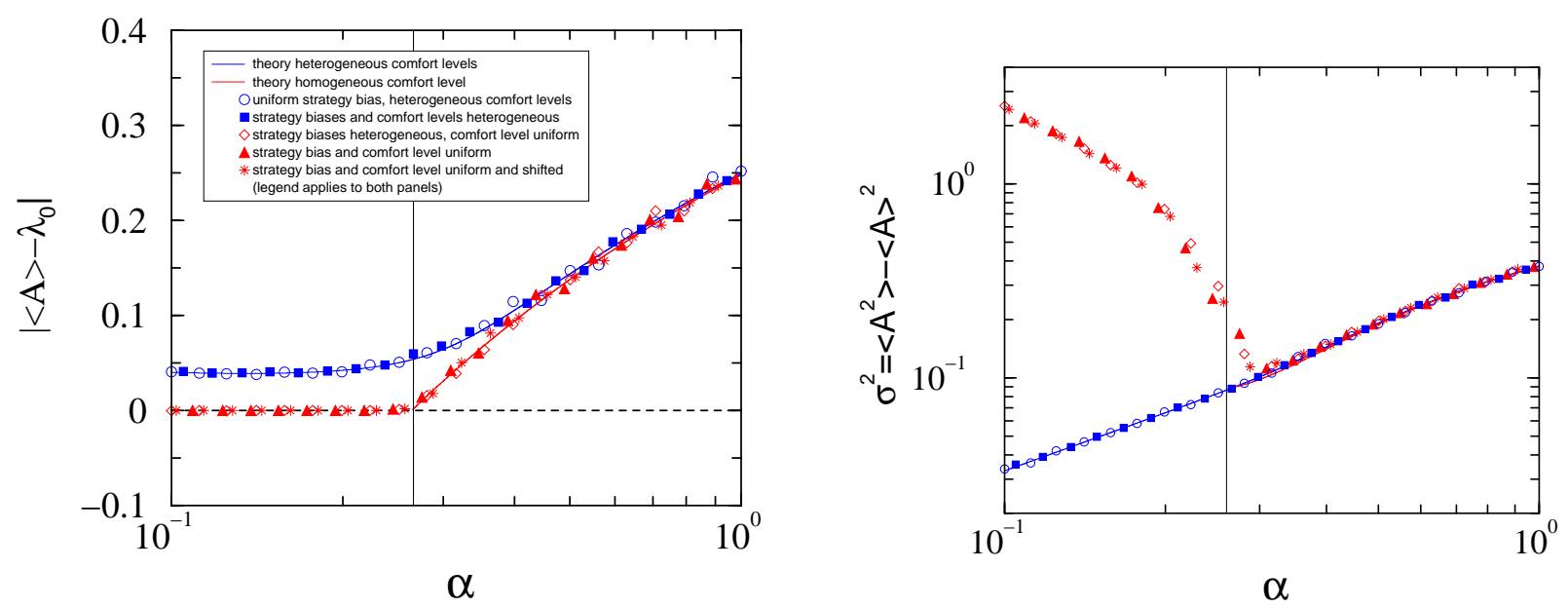

Figure 3. (Colour on-line) Deviation of the mean attendance $\langle A\rangle$ from the mean comfort level $\lambda_{0}$ (left), and fluctuations of the attendance (right). Solid lines are from theory, markers from simulations, unbiased starts, $N=1000$ agents, run for $10^{5}$ steps, averaged over 10 samples. Triangles refer to a system with uniform strategy bias and uniform comfort level $\left(\rho_{i} \equiv 0, \lambda_{i} \equiv 0.5\right)$, stars to the shifted case $\rho_{i} \equiv-0.5, \lambda_{i} \equiv 0$. Diamonds, circles and squares represent cases (i), (ii) and (iii) as detailed in the main text. Vertical lines mark the phase transition for the model with uniform comfort level $\lambda_{i} \equiv \lambda_{0}=0.5$ as found from the analytical theory.

in Fig. (3). Results are identical to those of (iv), confirming the translation invariance against simultaneous uniform shifts of the comfort levels and strategy biases.

\subsection{Fluctuating comfort levels}

Finally, we consider comfort levels which fluctuate in time in this section. Timedependent comfort levels present a form of disorder which is not quenched so that the standard analytical tools are not straightforwardly applicable to this case. All results presented in this section therefore rely on numerical simulations of the online process (11). Fluctuating comfort levels are here implemented by drawing each $\lambda_{i}=\lambda_{i}(t)$ from a bimodal distribution at every time step with no correlations in time $\left(\overline{\lambda_{i}(t) \lambda_{i}\left(t^{\prime}\right)}-\overline{\lambda_{i}(t)} \overline{\lambda_{i}\left(t^{\prime}\right)}=0\right)$. We here distinguish between collectively and individually fluctuating comfort levels,

$$
\begin{array}{lll}
\text { collectively fluctuating: } & \lambda_{i}(t) \equiv \lambda_{0}+\varepsilon \zeta(t), \\
\text { individually fluctuating: } & \lambda_{i}(t) \equiv \lambda_{0}+\varepsilon \zeta_{i}(t) .
\end{array}
$$

Here $\zeta(t)$ and the $\zeta_{i}(t)$ are drawn at random at every time step from the set $\{-1,1\}$ with equal probability, with no correlations over time (or between players in the individually fluctuating case). All strategy tables are generated at the beginning of the game, and remain fixed thereafter. We limit the discussion to the case of vanishing strategy biases 

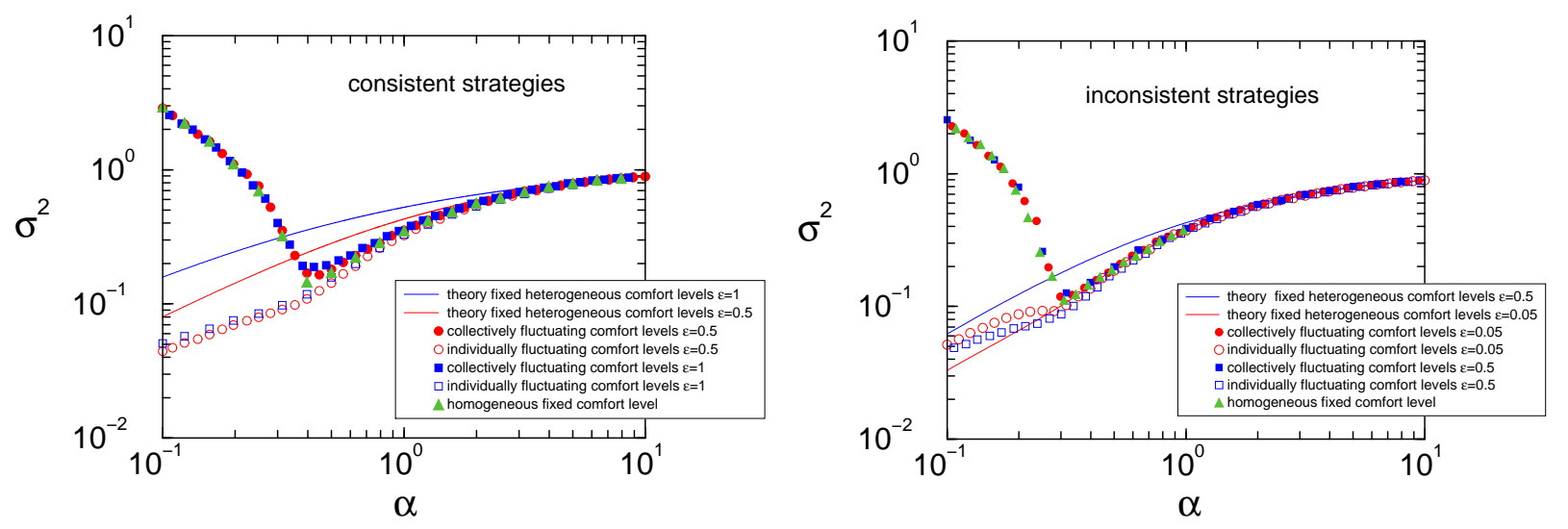

Figure 4. (Colour on-line). Game with resource levels fluctuating in time. Left: strategies consistent on average, right: inconsistent (see text for details). Simulations are for $N=300$ agents in the consistent case and $N=1000$ agents for inconsistent strategy assignments. Solid lines in both panels are for comparison only, and show the theoretical lines for heterogeneous comfort levels at same spread $\varepsilon$, but fixed in time. Filled triangles are simulations of the model with time-independent homogeneous comfort level.

$\left(\rho_{i}=0\right.$ for all $\left.i\right)$ and study models with consistent and with inconsistent strategies. Consistency here refers to consistency as a time-average, i.e. to $\lambda_{0}=\left\langle\lambda_{i}(t)\right\rangle=\rho_{0}=0$ for the consistent case and to $\lambda_{0} \neq 0$ otherwise.

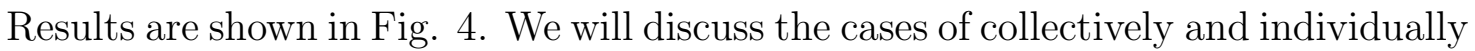
fluctuating comfort levels separately in the following, and focus on the volatility of the attendance.

4.3.1. Collectively fluctuating comfort levels: the corresponding volatilities are indicated as solid circles and squares in Fig. 目 with the consistent case shown in the left panel, and the inconsistent case in the right one. We show results for two different magnitudes $\varepsilon$ in each case. For comparison we also display the analytical curves for fixed comfort levels (at the same degree of inconsistency as the simulations with fluctuating levels) as triangles in both panels. One thus concludes that a collectively fluctuating comfort level does not cause any noticeable effect on the resulting volatility of the system, but that the system instead behaves as if the collective comfort level was fixed at its time-average.

4.3.2. Individually fluctuating comfort levels: the case of individually fluctuating comfort levels appears to be crucially different from the game with collectively modulated comfort level. Results are displayed as open markers in Fig. 4, and one finds that individually fluctuating comfort levels reduce the volatility significantly at small and intermediate values of $\alpha$ as compared to the system with homogeneously fixed 
or collectively fluctuating levels. At larger values of $\alpha$ no such effect is found. While we cannot fully control equilibration in our experiments, due to apparently increased relaxation times, our simulations seem to suggest that individually fluctuating levels reduce the volatility below the one observed in a system with fixed heterogeneous comfort levels of the same spread $\varepsilon$ (indicated as lines in Fig. 4 for comparison). In particular we find that after sufficiently long $\varepsilon$-dependent waiting times, the observed volatility shows only little sensitivity to the numerical value of $\varepsilon$.

\section{Geometrical interpretation of the phase transition}

Our findings regarding the absence of the phase transition in El-Farol models with heterogeneous comfort levels is interesting also from the point of view of statistical mechanics of the MG. In this final section we will discuss a geometrical interpretation of the phase transition of the original MG and will make some suggestions of how it may be possible to approach the problem of characterising MG models according to the presence or absence of the phase transition marked by a diverging integrated response.

The update rules of most known versions of the MG are of the form $q_{i}(t+1)=$ $q_{i}(t)+\xi_{i}^{\mu(t)} A^{\mu(t)}[\mathbf{q}(t)]$ (in the on-line formulation), with some global time-dependent quantity $A^{\mu(t)}[\mathbf{q}(t)]$ which depends on the information pattern $\mu(t)$ presented to the agents at $t$ and on the strategy score differences $\mathbf{q}(t)=\left(q_{1}(t), \ldots, q_{N}(t)\right)$ of all agents. A geometrical picture behind the transition of the standard MG can here be devised as follows [16, 17]: due to the above update rule, the $N$-dimensional vector $\mathbf{q}(t)$ will move in the space spanned by the $\alpha N$ vectors $\boldsymbol{\xi}^{\mu}=\left(\xi_{1}^{\mu}, \ldots, \xi_{N}^{\mu}\right), \mu=1, \ldots, \alpha N$. We will abbreviate this space by $V_{\alpha N}(\{\boldsymbol{\xi}\})=\left\{\sum_{\mu=1}^{\alpha N} c_{\mu} \boldsymbol{\xi}^{\mu} \mid c_{1}, \ldots, c_{\alpha N} \in \mathbb{R}\right\}$ in the following. Since a number $\phi N$ of agents can generally been shown to 'freeze' in MGs (where the fraction of frozen agents $\phi=\phi(\alpha)$ depends on the details of the model), i.e. to employ one strategy only and to have $\left|q_{i}(t)\right| \rightarrow \infty$ in the long-time limit, the effective number of degrees of freedom is reduced to $[1-\phi(\alpha)] N$, so that $\mathbf{q}(t)$ can be thought of as having $[1-\phi(\alpha)] N$ free components and moving in the $\alpha N$-dimensional vector space $V_{\alpha N}(\{\boldsymbol{\xi}\})$. If $\alpha>1-\phi(\alpha)$ then any perturbation on $\mathbf{q}(t)$ can be washed out by the dynamics. If however $\alpha<1-\phi(\alpha)$ this may not be the case (as the dynamics is restricted to movements in $V_{\alpha N}(\{\boldsymbol{\xi}\})$ ), and initial conditions may become relevant. The point at which ergodicity breaking occurs (and the integrated response diverges) can thus be identified as $\alpha_{c}=1-\phi\left(\alpha_{c}\right)$, which is indeed fulfilled at the transition point of the standard MG, as illustrated in the inset of Fig. 5 .

This picture breaks down whenever the update rules are not of a form which moves the vector $\mathbf{q}(t)$ in the space spanned by the $\left\{\boldsymbol{\xi}^{\mu}\right\}$, specifically if $\mathbf{q}(t+1)-\mathbf{q}(t)$ is not a linear combination of the $\left\{\boldsymbol{\xi}^{\mu}\right\}$, and one might not necessarily expect to see a transition with diverging integrated response in this case. Several examples can be listed here. In grand-canonical MGs [10] one has update rules of the (schematic) form

$q_{i}(t+1)-q_{i}(t)=-\xi_{i}^{\mu(t)} A^{\mu(t)}-\kappa$, with a model parameter $\kappa$, and the transition is absent as soon as $\kappa \neq 0$. MGs with finite score memory [11] have update rules of the form 


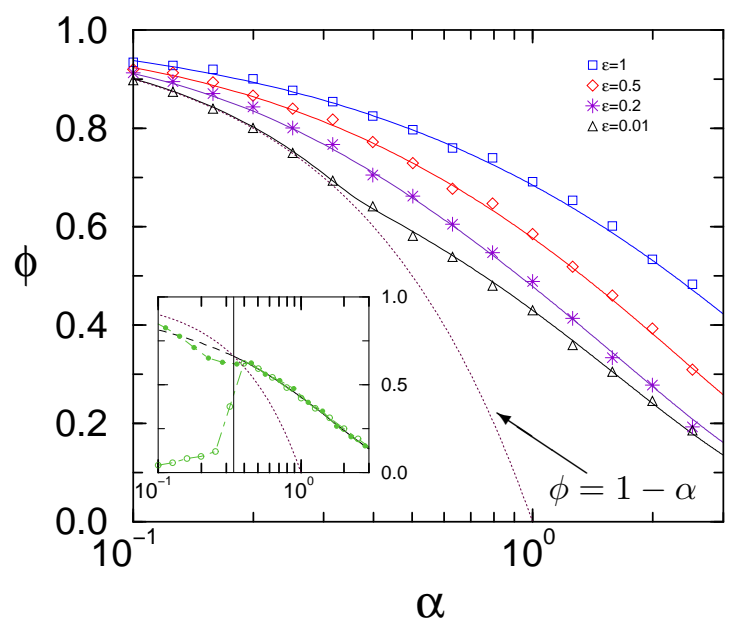

Figure 5. (Colour on-line) Fraction of frozen agents $\phi$ as a function of $\alpha$. Main panel: model with consistent strategies, and heterogeneous fixed comfort levels $\left(\rho_{i} \equiv 0 \forall i\right.$ and $\lambda_{i}= \pm \varepsilon$ randomly with $\varepsilon=1,0.5,0.2,0.01$ from top to bottom. Symbols are from simulations of the batch process $(N=300$ agents, averages over 10 samples, run for a minimum of 1000 batch steps, and longer if required for equilibration). Solid lines are predictions of the analytical theory. Inset: standard MG $(\varepsilon=0)$, open symbols represent simulations with tabula rasa initial conditions, full symbols biased starts. Vertical line in the inset marks $\alpha_{c}$ where $\alpha_{c}=1-\phi\left(\alpha_{c}\right)$.

$q_{i}(t+1)-q_{i}(t)=-\gamma q_{i}(t)-\xi_{i}^{\mu(t)} A^{\mu(t)}$ (with $0 \leq \gamma \leq 1$ defining the inverse time-scale over which scores are forgotten), and again the transition seems absent as soon as $\gamma>0$. No analytical results are available for games with finite score memory though. Finally the transition marked by a diverging integrated response is also absent in MGs with impact correction and with dilution, but instead preceded by a memory-onset transition (at which $\chi$ remains finite). In both cases one has $q_{i}(t+1)-q_{i}(t)=-\xi_{i}^{\mu(t)} A_{i}^{\mu(t)}$ where $A_{i}^{\mu(t)}$ carries an explicit dependence on $i$, hence invalidating the above picture of $\mathbf{q}(t)$ moving in $V_{\alpha N}(\{\boldsymbol{\xi}\})$. Specifically $A_{i}^{\mu}=A^{\mu}(t)-(\nu / \sqrt{N}) \xi_{i}^{\mu} s_{i}(t)$ in the model with impact correction (with $\nu$ measuring the ability of the agents to correct for their own impact), and $A_{i}^{\mu}(t)=N^{-1 / 2} \sum_{j}\left(c_{i j} / c\right)\left\{\omega_{j}^{\mu}+\xi_{j}^{\mu} s_{j}(t)\right\}$ in the model with dilution (where $c_{i j} \in\{0,1\}$ and $\left.P\left(c_{i j}=1\right)=c\right)$.

In the model studied in this paper model one has $A_{i}^{\mu}=A^{\mu}-\lambda_{i}$ with $\lambda_{i}$ the comfort level of player $i$. Thus the quantity $A_{i}$ does not depend on $i$ if $\lambda_{i} \equiv \lambda_{0}$ and the above geometric interpretation holds and one is not surprised to find the typical MG-transition. For heterogeneous $\left\{\lambda_{i}\right\}$ this is no longer the case, the picture of the vector $\mathbf{q}(t)$ moving in $V_{\alpha N}(\{\boldsymbol{\xi}\})$ breaks down and the transition is absent.

Having the listed examples in mind, one may thus speculate whether the property of the update rules of the standard MG of restricting the increments of $\mathbf{q}(t)$ to $V_{\alpha N}(\{\boldsymbol{\xi}\})$ is a necessary condition for a phase transition with diverging integrated response to occur. Note that such a dynamics would probably not be a sufficient condition for this type of 
transition to be present as additionally the condition $\alpha=1-\phi(\alpha)$ has to be met at $\alpha_{c}$. Interestingly we find that in our model with heterogeneous comfort levels this condition is never fulfilled, as $\phi(\alpha)>1-\alpha$ for all tested values of $\alpha$ (see Fig. 5). Only as the degree of heterogeneity $\varepsilon$ approaches zero we do find that $\phi(\alpha) \rightarrow 1-\alpha$ for $\alpha<\alpha_{c}$ (where $\alpha_{c}$ is the transition point of the MG with uniform comfort level, $\varepsilon=0$ ). Similar observations can be made for the GCMG. In order to test our hypothesis further, one may want to look for a model in which the dynamics is not restricted to $V_{\alpha N}(\{\boldsymbol{\xi}\})$, but in which there is an $\alpha$ so that $1-\phi(\alpha)=\alpha$. The MG with finite score memory might here be a candidate as no frozen agents are present at any $\alpha$ after long-enough equilibration [11. Unfortunately no analytical information on its phase behaviour is available at present.

\section{Concluding remarks}

We have investigated a mathematical formulation of the El-Farol bar problem focusing on the effects of heterogeneity in the comfort levels and/or the biases of the agents' strategies. Generalizing earlier results of [8] our main finding consists in the observation that heterogeneity in the comfort levels even of infinitesimal degree removes the phase transition of the standard MG, while no such removal is observed for heterogeneous strategy biases. This transition has also been shown to be present in an El-Farol bar problem with homogeneous comfort level. There one finds a phase at low but non-zero inconsistency of the strategies, in which the mean attendance converges to the comfort level, and a second phase in which the comfort level cannot be retrieved due to too large an inconsistency in the strategy vectors. In the phase of successful retrieval fluctuations of the attendance around the comfort level are large (for tabula rasa starts), but depend on initial conditions. In the model with heterogeneous (i.e. agent-dependent) comfort levels the attendance converges to the mean comfort level if and only if strategies are fully consistent. This property is lost in the presence of heterogeneous comfort levels for

any degree of inconsistency at any finite $\alpha$. At the same time models with heterogeneous comfort levels appear to show low fluctuations of the attendance, and the typical highvolatility branches of the model with homogeneous levels are absent. Most interestingly heterogeneous strategy biases at uniform comfort levels do not have the same effect, the transition is preserved. Hence we can strictly trace removal of the transition back to the spread in comfort levels, and find that the equivalence of comfort levels and strategy biases of the homogeneous case does not carry over to the heterogeneous one.

We have also studied El-Farol games with temporally fluctuating comfort levels and find that the behaviour of the volatility remains unchanged if the level fluctuates collectively for all players. Individually fluctuating levels, however, can reduce the volatility significantly.

Finally we have pointed out the similarities of the present model with other MGtype systems with and without phase transitions, and have discussed a geometrical interpretation of the updated rules, which may allow to characterise MG-models 
according to the presence or otherwise of the ergodicity breaking transition marked by a singular integrated response. We hope that these suggestions may stimulate further investigation of such models with a focus on their classification according to the types of their respective phase transitions.

\section{Acknowledgements}

This work was supported by the European Community's Human Potential Programme under contract HPRN-CT-2002-00319, STIPCO and by EVERGROW, integrated project No. 1935 in the complex systems initiative of the Future and Emerging Technologies directorate of the IST Priority, EU Sixth Framework. TG would like to thank David Sherrington for fruitful discussions.

\section{References}

[1] Challet D, Marsili M and Zhang Y-C 2005 Minority Games (Oxford University Press, Oxford UK)

[2] Coolen A C C 2005 The Mathematical Theory of Minority Games (Oxford University Press, Oxford UK)

[3] Johnson N F, Jefferies P and Hui PM 2003 Financial market complexity (Oxford University Press, Oxford UK)

[4] Arthur W B 1994 Amer. Econ. Assoc. Papers Proc. 84406

[5] Challet D, Zhang Y-C 1997 Physica A 246407

[6] Johnson N F, Hui P M , Zheng D, Tai C W 1999 Physica A 269493

[7] Yip K F, Hui P M, Lo T S, Johnson N F 2003 Physica A 321318

[8] Challet D, Marsili M, Ottino G 2004 Physica A 332469

[9] Challet D 2004 Physica A 34424

[10] Challet D, Marsili M 2003 Phys. Rev. E 68036132

[11] Challet D, De Martino A, Marsili M, Perez Castillo I 2006 JSTAT P03004

[12] De Martino A, Marsili M 2001 J. Phys. A: Math. Gen. 342525

[13] Galla T 2005 JSTAT P01002

[14] Heimel J A F and Coolen A C C 2001 Phys. Rev. E 63056121

[15] Heimel J A F, De Martino A 2001 J. Phys. A: Math. Gen. 34 L539

[16] Heimel J A F $2001 \mathrm{PhD}$ thesis King's College London

[17] Marsili M, Challet D 2001 Phys. Rev. E 64056138

\section{Appendix: Equations describing the ergodic stationary states}

Assuming time-translation invariance (i.e. $C_{t t^{\prime}}=C\left(t-t^{\prime}\right)$ and similarly for $G_{t t}$ and $\left.G_{t t^{\prime}}^{\prime}\right)$ and finite integrated response one follows the standard ansatz to proceed from the effective agent problem to explicit equations characterising the relevant persistent order parameters of the ergodic stationary states. In our problem these are given by $c$, the persistent part of the correlation function, and by $\chi=\sum_{\tau} G(\tau)$ and $\chi^{\prime}=\sum_{\tau} G^{\prime}(\tau)$. Further details on this analysis relying on a separation of so-called fickle and so-called frozen agents can be found in [2]. 
The resulting $3 \times 3$ system of non-linear equations for $\left\{c, \chi, \chi^{\prime}\right\}$ then reads

$$
c=\int d \lambda R(\lambda) \widetilde{c}(\lambda), \quad \chi=\int d \lambda R(\lambda) \widetilde{\chi}(\lambda), \quad \chi^{\prime}=\int d \lambda R(\lambda) \lambda \widetilde{\chi}(\lambda)
$$

with $R(\lambda)$ the distribution from which the strategy biases $\lambda_{i}$ are drawn and where $\widetilde{\chi}(\lambda)$ and $\widetilde{c}(\lambda)$ given by

$$
\begin{gathered}
\tilde{\chi}(\lambda)=\frac{(1+\chi)}{\alpha} \operatorname{erf}\left(\frac{\sqrt{\alpha}}{\sqrt{2 g(\lambda)}(1+\chi)}\right), \\
\widetilde{c}(\lambda)=1-\operatorname{erf}\left(\frac{\sqrt{\alpha}}{\sqrt{2 g(\lambda)}(1+\chi)}\right)+\left[\frac{(1+\chi)^{2} g(\lambda)}{\alpha}\right] \\
\times\left\{\operatorname{erf}\left(\frac{\sqrt{\alpha}}{\sqrt{2 g(\lambda)}(1+\chi)}\right)-\sqrt{\frac{2 \alpha}{\pi g(\lambda)(1+\chi)^{2}}} \exp \left(-\frac{\alpha}{2 g(\lambda)(1+\chi)^{2}}\right)\right\},
\end{gathered}
$$

and where $g(\lambda)$ is the persistent part of the temporal correlations of the noise $\eta_{\lambda}(t)$ in the effective agent problem which reads

$$
g(\lambda)=\frac{1+c}{(1+\chi)^{2}}+2 \frac{\left(\rho_{0}+\chi^{\prime}\right)^{2}}{(1+\chi)^{2}}-4 \lambda \frac{\rho_{0}+\chi^{\prime}}{1+\chi}+2 \lambda^{2} .
$$

Following [2] the fluctuations of the attendance can be approximated as

$$
\left\langle A^{2}\right\rangle-\langle A\rangle^{2}=\frac{1}{2} \frac{1+c}{(1+\chi)^{2}}+\frac{1}{2}(1-c),
$$

and the mean attendance level turns out to be

$$
\langle A\rangle=\frac{\rho_{0}+\chi^{\prime}}{1+\chi} .
$$

